\title{
Optimization of experimental snowflake configurations on TCV
}

\author{
R. Ambrosino ${ }^{1}$, R. Albanese ${ }^{2}$, S. Coda $^{3}$, M. Mattei ${ }^{4}$, J.-M. Moret ${ }^{3}$, H. Reimerdes ${ }^{3}$
}

\begin{abstract}
The design of a snowflake (SF) equilibrium requires a strong effort on the poloidal field (PF) currents in terms of MAturns and mechanical loads. This has limited the maximum plasma current in SF configurations on Tokamak à Configuration Variable (TCV) to values well below the intrinsic MHD limits. In this paper the definition of optimized snowflake (SF) configurations in TCV and their experimental tests are illustrated. The PF current optimization procedure proposed in [1] is adapted and applied to a snowflake scenario in TCV where the PF currents were close to their operational limits with the aim of reducing the total MAturns in view of higher values of the plasma current. This procedure optimizes the PF currents while fulfilling the machine technological constraints for a given bound on the tolerable plasma shape changes. The method exploits the linearized relation between the plasma-wall gaps and the PF currents. In the investigated TCV scenario the optimization procedure allowed a $20 \%$ increase of the plasma current while keeping the plasma shape alignment with respect to the nominal shape within a tolerance of $1 \mathrm{~cm}$. The predicted optimization potential was confirmed in a TCV experiment.
\end{abstract}

Keywords: Poloidal field system, snowflake divertor configuration, tokamak.

\section{INTRODUCTION}

The European roadmap to fusion energy has to face a number of technical challenges, for which eight different missions have been defined [2]. Mission 2 "Heat-exhaust systems" addresses the challenge of reducing the heat load on the divertor targets. The fusion community is currently assessing several alternatives to the conventional divertor, including the 'Advanced Magnetic Configurations' such as Snowflake (SF) [3] and Super-X [4]. Snowflake configurations are characterized by a hexapole magnetic field, i.e. potentially four divertor legs compared to the usual two, and accompanied by a larger low field region than in a conventional quadrupole divertor. These configurations are able to flare the scrape-off layer and increase the magnetic connection length, thus reducing the peak heat load and favouring detached divertor conditions.

Promising experimental results on advanced configurations have been obtained in TCV [5], [6], DIII-D [7] and NSTX [8]-[9], and the possibility to create snowflake configurations

$1 \mathrm{R}$. Ambrosino is with Consorzio CREATE and the Dipartimento di Ingegneria (DI), Università degli Studi di Napoli Parthenope, Napoli, Italy. email: ambrosino@uniparthenope.it

2 R. Albanese is with Consorzio CREATE and the Dipartimento di Ingegneria Elettrica e Tecnologie dell'Informazione (DIETI), Università degli Studi di Napoli Federico II, Napoli, Italy

3 S. Coda, J.-M. Moret and H. Reimerdes are with Ecole Polytechnique Fèdèrale de Lausanne (EPFL), Centre de Recherches en Physique des Plasmas, CH-1015 Lausanne, Switzerland

$4 \mathrm{M}$. Mattei is with Consorzio CREATE and the Dipartimento di Ingegneria Industriale e dell'Informazione (DIII), Seconda Università di Napoli, Aversa (CE), Italy has been considered in EAST for the experimental campaign 2014 [10]. The first attempts to determine such new plasma configurations have revealed the inherent difficulty to obtain them with acceptable coil currents.

A possible strategy to design snowflake configurations with reduced PF coil currents have been proposed in [1]. The aim of the present paper is to show an application of these methods and techniques in TCV experiments. A TCV snowflake scenario achieved in a previous pulse has been optimized with the objective of increasing the plasma current while satisfying the operational constraints and keeping the perturbation of the plasma shape within a given tolerance.

The optimization of the poloidal field (PF) currents was tested on pulse 47651 [11] where the PF currents were close to their operational limits. The optimization allowed a $20 \%$ increase of the plasma current while keeping the plasma shape, defined by the magnetic separatrix around the core plasma, aligned with respect to the nominal 47651 shape within a tolerance of few $\mathrm{cm}$. An experimental validation on pulse 49626 confirmed the calculations with an increase of plasma current limited to $6 \%$ for safety reasons. Since the experiment showed a good agreement with the numerical simulations, the predicted increase by $20 \%$ should also be attainable.

\section{TCV MODEL}

The SF design and optimization procedure in [1] requires free boundary equilibrium calculations and linearized MHD equilibrium models that describe the general response of the plasma to changes in the PF coil currents. In this section the models for TCV plasma configurations generated using the CREATE-NL and CREATE-L simulation codes [12] are illustrated. CREATE-NL is a nonlinear equilibrium code, from which the linearized model of the plasma response is evaluated numerically with a small variation method with respect to the initial equilibrium configuration. On the other hand, the CREATE-L response model is obtained analytically by derivation of the Grad-Shafranov equation.

TCV active and passive structures [13] are modeled in the CREATE-NL and CREATE-L codes with (see Figure 1)

- $2 \mathrm{OH}$ circuits: independent ohmic circuits used to induce the plasma current

- $8 \mathrm{E}$ and $8 \mathrm{~F}$ shaping coils connected to 16 independent power supplies

- FPS circuit: in vessel circuits used for the vertical stability (G1-6 coils)

- BCOIL circuit: used to produce toroidal field, it is composed by 16 coils surrounding the vacuum vessel and connected together in series by bus bar connections 


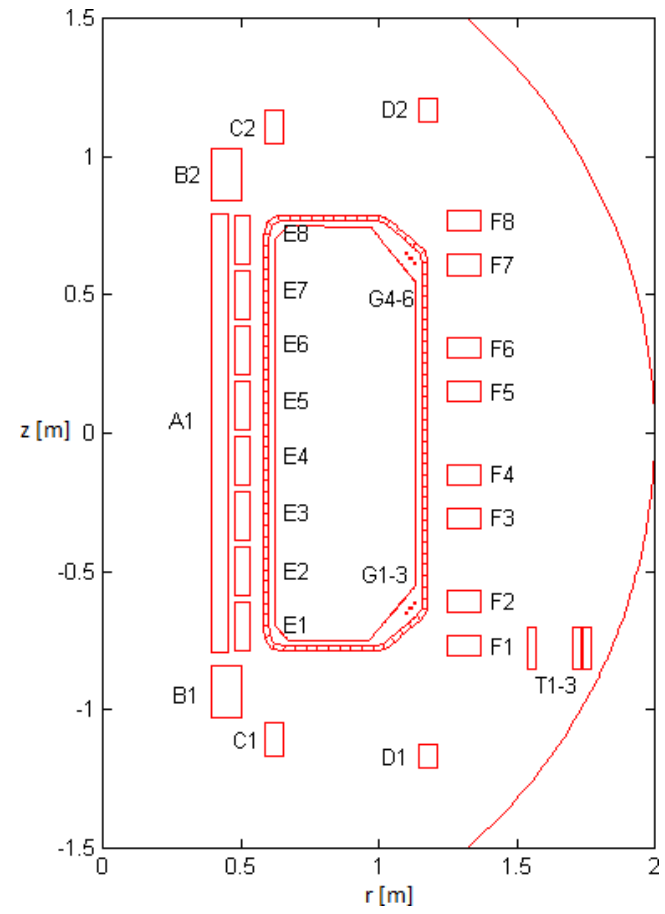

Fig. 1. Poloidal field system of TCV. The $O H 1$ current flows in the central solenoid $A 1$ whereas the $O H 2$ circuit has the $B 1, B 2, C 1, C 2, D 1, D 2$ coils in series.

whose poloidal field is approximated by the coils T1,

$\mathrm{T} 2$ and $\mathrm{T} 3$ connected in series [13].

- vacuum vessel

- 38 flux loops

- 38 pick-up coils.

The validity of our models has been tested on TCV experimental data with and without plasma.

\section{A. Tests on the model without plasma}

The static and dynamic response of the magnetic measurements to OH1-2, E1-8 and F1-8 currents has been tested in a set of $19 \mathrm{TCV}$ pulses without plasma. Using the models without plasma it was verified that the effect of PF coil current variations on the magnetic measurements variation was predicted with a relative accuracy of approximately $5 \%$ with a good agreement between CREATE-L and CREATENL models.

Figure 2 shows the prediction average relative error obtained in correspondence with PF circuit/coil current variations (18 in total ordered as circuits OH1-2, coils E1-8 and F1-8) on flux loops and pick up coils.

The effect of passive currents on the dynamics without plasma is modeled with the following Linear Time Invariant (LTI) system

$$
L_{v} \frac{d I_{v}}{d t}+R_{v} I_{v}=-M_{v a} \frac{d I_{a}}{d t}
$$

where $I_{a}$ is the set of active currents in $O H, E, F$ and $G$ circuits, $I_{v}$ refers to a set of 100 discrete currents approximating the passive current distribution in the vessel, $R_{v}$ is the resistance matrix of the passive structures, whereas
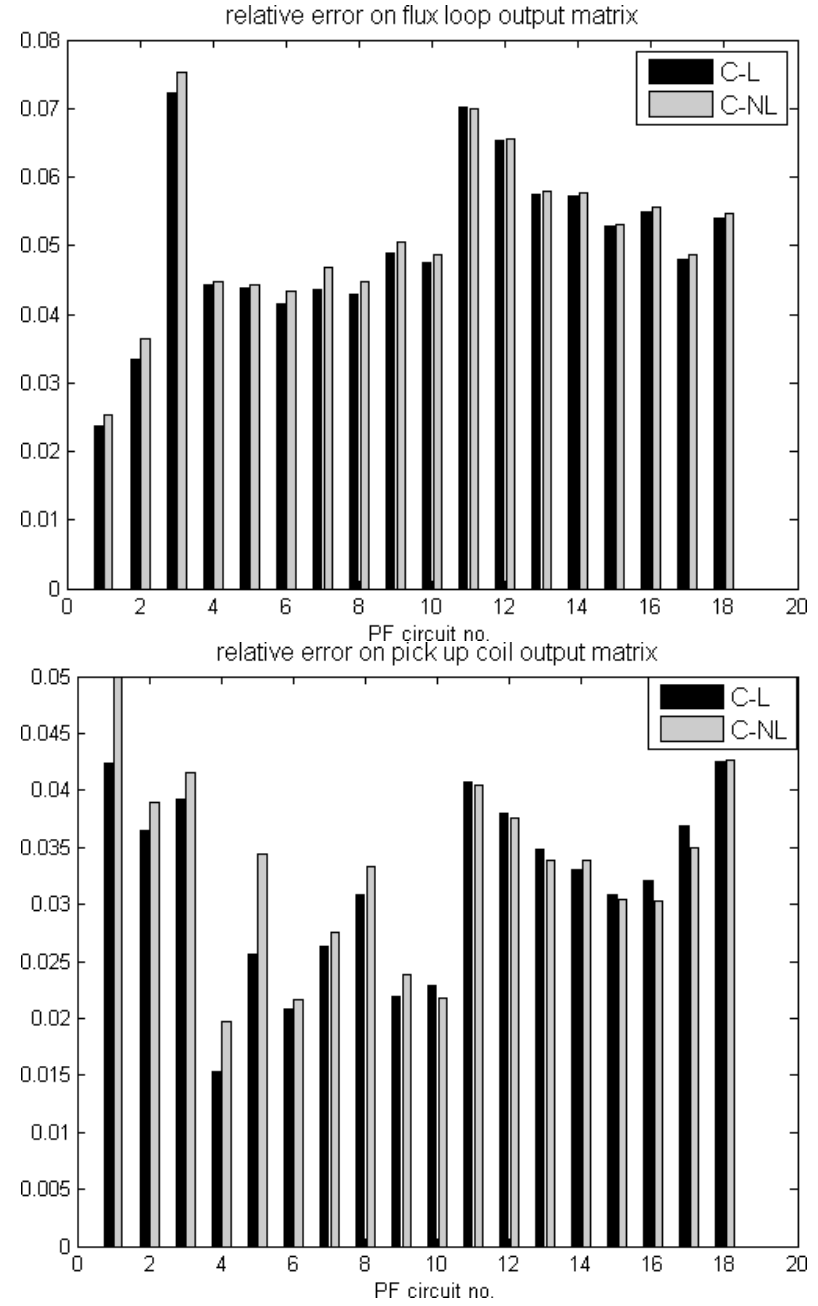

Fig. 2. Average prediction relative error on the magnetic measurements to each one of the $18 \mathrm{PF}$ circuit/coil currents (ordered as circuits OH1-2, coils E1-8 and F1-8) with both CREATE-L and CREATE-NL models

$L_{v}$ and $M_{v a}$ are the auto and mutual inductance matrices. To eliminate the dependence on the derivative of the active currents representing an input for the evolution of the eddy currents in the passive structures, an auxiliary variable $W_{v}$ is introduced, which is the time integral of $I_{v}$, yielding:

$$
L_{v} \frac{d W_{v}}{d t}+R_{v} W_{v}=-M_{v a} I_{a}+M_{v a} I_{a}(0)+L_{v} I_{v}(0)
$$

and hence:

$$
I_{v}=-L_{v}^{-1}\left(R_{v} W_{v}+M_{v a} I_{a}-M_{v a} I_{a}(0)-L_{v} I_{v}(0)\right) .
$$

The magnetic measurements are obtained by means of a linear combination of both the active circuit and eddy currents. Figure 3 shows that the eddy current effects are properly taken into account. The presence of the vessel passive structures, which have the slowest time constants of 12.4 and $6.8 \mathrm{~ms}$, cause a dynamic delay in the magnetic measurements response to the active currents variation which is well predicted by our linear model. 

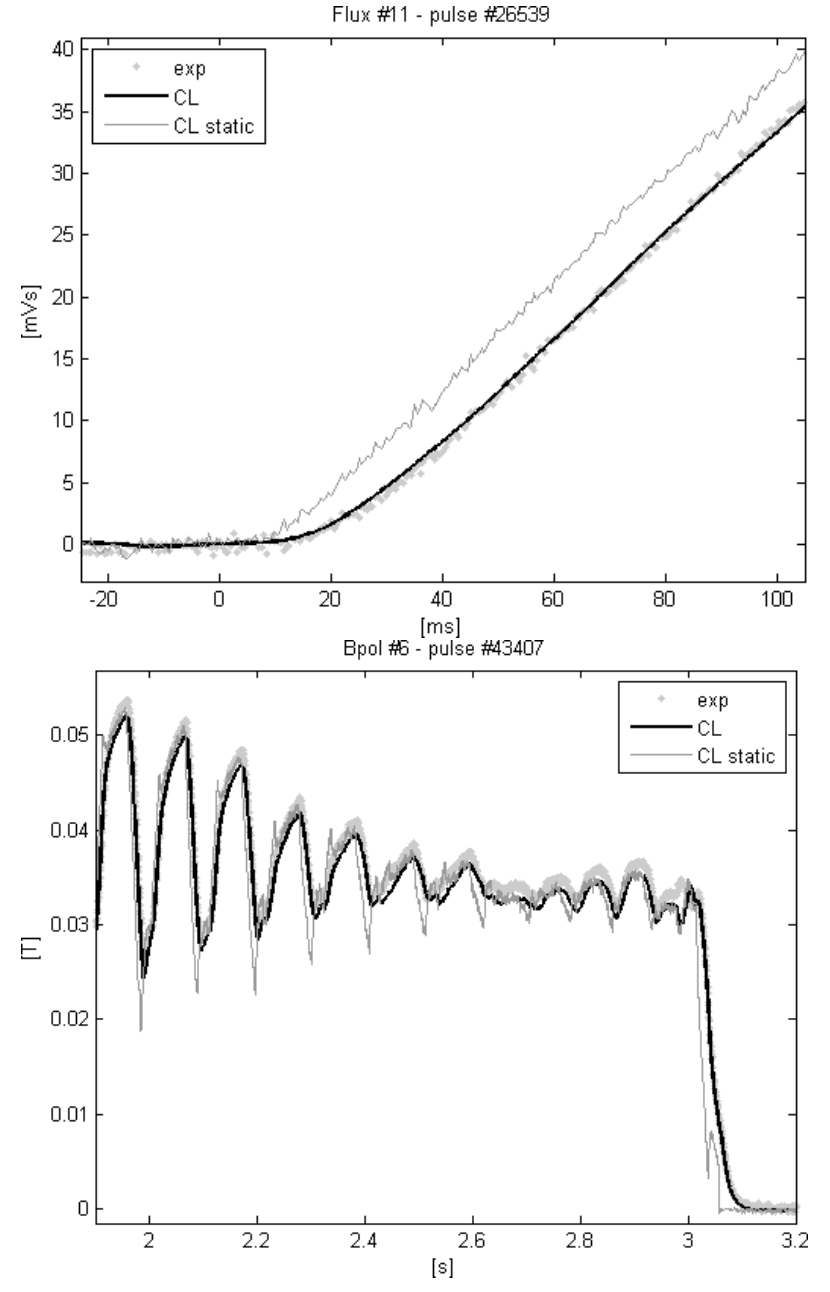

Fig. 3. Comparison of static and dynamic CREATE-L models with the experimental data. Flux probe \#11 in pulse 26539 (F7 excitation) and pick-up \#1 in standard dry run 43407.

\section{B. Tests on the model with plasma}

A plasma equilibrium depends on the value of the active currents but also on the plasma current density profile and on the eddy currents in the vessel. The plasma current profile parameterization described in [12] is assumed in the present study with the parameter values selected so as to fit the plasma current $I p$, the poloidal beta $\beta_{p o l}$ and the internal inductance $l_{i}$ provided by the TCV magnetic reconstruction system.

In order to provide a model validation, different TCV experimental equilibria has been evaluated with CREATE$\mathrm{NL}$ and compared with the reconstruction estimates obtained with the LIUQE equilibrium code [14]. The following limited, diverted and a snowflake plasma equilibrium reconstructions have been considered:

- Limited equilibrium: plasma pulse 9482 at $0.45 \mathrm{~s}$ $\left(\mathrm{Ip}=235 \mathrm{kA}, \beta_{p o l}=0.16, l_{i}=1.19\right)$. The deformable MHD-consistent CREATE-L plasma response model provides a growth rate of $282 s^{-1}$ and a stability margin $m_{s}=0.89$.

- Single null equilibrium: plasma pulse 47655 at $0.8 \mathrm{~s}$

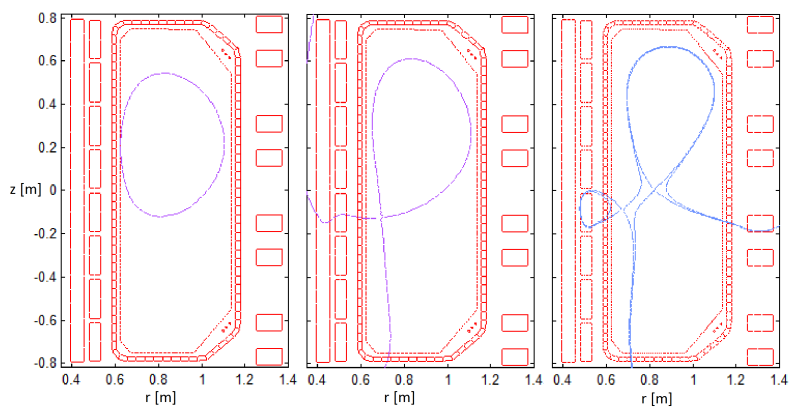

Fig. 4. Plasma boundaries of the pulses 9482 at $0.45 \mathrm{~s}$ (left), 47651 at $0.7 \mathrm{~s}$ (center) and 47655 at $0.8 \mathrm{~s}$ (right).

$\left(\mathrm{Ip}=255 \mathrm{kA}, \beta_{\text {pol }}=0.28, \quad l_{i}=1.21\right)$. The deformable MHD-consistent CREATE-L plasma response model provides a growth rate of $618 \mathrm{~s}^{-1}$ and a stability margin $m_{s}=0.41$ as defined in [15].

- Snowflake equilibrium: plasma pulse 47651 at $0.7 \mathrm{~s}$ $\left(\mathrm{Ip}=275 \mathrm{kA}, \beta_{\text {pol }}=0.36, l_{i}=1.12\right)$. The deformable MHD-consistent CREATE-L plasma response model provides a growth rate of $1961 \mathrm{~s}^{-1}$ and a stability margin $m_{s}=0.14$.

The plasma boundaries of the equilibria in the three cases are illustrated in Figure 4. In all the cases the magnetic measurements are reproduced with a relative error of about $5 \%$ and the plasma boundary is predicted with an accuracy of the order of $1 \mathrm{~cm}$ [16] with respect to the LIUQE reconstruction.

C. Experimental validation of the vertical instability modeling

Snowflake configurations are characterized by a large elongation since the separatrix legs cross at angles of 60 degrees instead of the usual 90 of a single null plasma. For this reason, particular attention has been paid to the estimation of the growth rate of the vertical instability so as to remain within the capabilities of the feedback stabilization system. The growth rate is computed as the unstable eigenvalue of the dynamic matrix of the linearized plasma response to active and passive currents [12]. In order to validate the growth rate prediction, the uncontrolled VDE (Vertical Displacement Event) occurring during the pulse 9482 (see Section II-B) has been considered. In principle all magnetic measurements should approach an exponential behavior when the vertical control is lost. However this is masked by different factors:

- the stable modes are not negligible in the first phase of the VDE;

- the model becomes highly nonlinear when the plasma hits the wall and disrupts;

- there are several sources of process and measurement noise.

On the selected pulse, the time evolution of the magnetic field measurements $B_{\text {pol }}(t)$ in the time interval from $t_{0}=0.455 \mathrm{~s}$ (trigger of the VDE) to $t_{\max }=0.480 \mathrm{~s}$ (beginning of the disruption phase) has been considered. A reduction of the noise is obtained taking the linear combination of the 38 field measurements corresponding to their first principal 


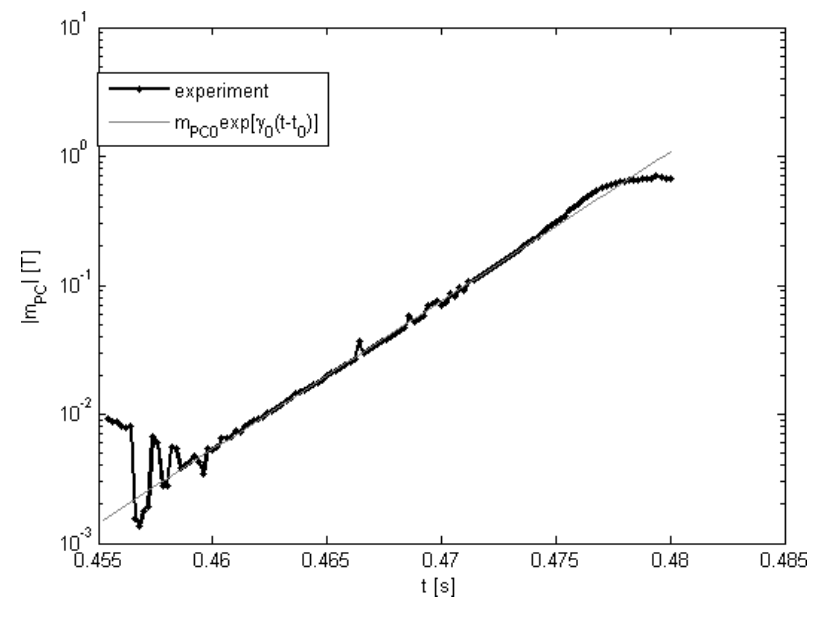

Fig. 5. Experimental behavior of $m_{P C}(t)$ during the uncontrolled vertical displacement event occurring in pulse 9482 using a logarithmic y-axis.

component whose evolution $m_{P C}(t)$ is reported in Figure 5. This figure shows that the experimental value of the growth rate $\left(265 \mathrm{~s}^{-1}\right)$ is in good agreement with the estimate provided by the model.

\section{OPTIMIZATION OF THE PF COILS CURRENTS OF A SNOWFLAKE CONFIGURATION}

\section{A. General snowflake figure of merits}

The optimization of a snowflake equilibrium can be done with respect to different figures of merit as

1) total ampere-turns in the active coils (sum of the absolute values) $I_{t o t}$

2) maximum active coil current normalized to its limit $\max \left\|I / I_{\text {lim }}\right\|$

3) maximum vertical forces on coils normalized to its limit $\max \left\|F_{z} / F_{z l i m}\right\|$

4) plasma volume $V_{p l}$;

5) minimum connection length $l_{c}$ within the Scrape-Off Layer (SOL);

6) SOL volume $V_{S O L}$;

7) flux expansion $F$;

8) distance between X-points of quasi snow flake (QSF) configurations $\sigma_{x}$

9) angle with X-axis of the line connecting the X-points of QSF configurations $\theta_{x}$

10) strike point positions

The first three quantities are related to the effort of the PF coil system in terms of current and vertical forces while the others consider the fusion power and the heat exhaust properties of the configuration.

\section{B. SF optimization in TCV: problem definition}

The SF optimization in TCV has been performed starting from the configuration of pulse 47651 at $0.8 \mathrm{~s}$ [11] where some of the PF currents and vertical forces were close to the operational limits (only $3.6 \%$ below). ${ }^{1}$

The aim of the optimization has been the design of a snowflake configuration at a higher plasma current than in pulse 47651 by means of a minimization of the PF coil related quantities (currents and forces) keeping the plasma shape as close as possible to the reference shape. Further constraints have been added on the growth rate (which for the nominal configuration is about $2000 s^{-1}$ ) and on the variation of the PF currents with respect to their reference value.

To achieve this result, the snowflake optimization procedure proposed in [1] has been adapted to the TCV problem assuming current and force constraints prescribed in [17]. These constraints are linear or quadratic functions of E- and F-coil currents, whose absolute values have to be below a limit and correspond to estimates of forces on the Fcoil supports and A- B- and E-coil assembly. The distance between the constrained currents and forces and their limits has been described by means of a so-called safety factor on the constraints $q_{c}=\left(\max \left\|s_{i}(I) / s_{\text {ilim }}\right\|\right)^{-1}, s_{i}(I)$ being the of value (function of the PF currents) of the $i-t h$ current or force constraint in [17]. The problem of increasing the plasma current for the optimized equilibrium can be then turned out into the maximization of the safety factor $q_{c}$.

Moreover the following machine constraints on the plasma separatrix have been added:

- the inboard gap at the equatorial plane greater than $0.05 \mathrm{~m}$ to avoid shadowing for heat and particle flux measurements at the HFS strike point;

- the lower strike point radial position at $0.73 \mathrm{~m}( \pm 0.01 \mathrm{~m})$ to optimize the configuration for heat and particle flux measurements at the lower strike point.

In addition $\mathrm{OH} 1$ and $\mathrm{OH} 2$ have been kept fixed to their experimental values as they are the sources of Ohmic flux during the discharge.

\section{SF optimization in TCV: simulation results}

In this section we firstly describe the ad hoc optimization procedure for TCV snowflake configuration that takes into account all the previous constraints; then the simulation results on the optimization of pulse 47651 at $0.8 \mathrm{~s}$ will be presented.

Let us consider an initial SF equilibrium, namely $E Q_{\text {init }}$, and indicate with $\overline{\mathbf{I}}_{\mathbf{P F}}$ the E-F currents on the active coils. The plasma boundary can be described by plasma-wall distances $\mathbf{g}$, which are called gaps, whose variation $\delta \mathbf{g}$ with respect to the PF currents is generally not linear [1]. However, a linearization can be considered [12] if the plasma boundary changes are small:

$$
\delta \mathbf{g}=\mathbf{C}_{\mathbf{G}} \delta \mathbf{I}_{\mathbf{P F}} .
$$

Indicating with

- $\mathbf{c}_{\mathbf{G e}}$ the row of the $\mathbf{C}_{\mathbf{G}}$ matrix related to the inboard gap variation at the equatorial plane,

${ }^{1}$ This pulse was obtained using the free-boundary equilibrium code FBTE [18] which considers the total Ampere-turns in its cost function as well as the costs of dipoles in adjacent coils, but not the exact form of the protection equations of TCV. 


\begin{tabular}{|c|c|c|c|c|}
\hline & $\Delta b[\mathrm{~cm}]$ & $\Delta I[\mathrm{kA}]$ & $\gamma\left[\mathrm{s}^{-1}\right]$ & $q_{c}$ \\
\hline Equil 'C1' & 0.2 & 0.75 & 1200 & 1.09 \\
\hline Equil 'C2' & 1 & 2 & 1200 & 1.20 \\
\hline
\end{tabular}

TABLE I

MAXIMUM BOUNDARY DISPLACEMENT $\Delta b$, MAXIMUM VARIATION OF THE E/F CURRENTS $\Delta I$, GROWTH RATE $\gamma$ AND SAFETY FACTOR $q_{c}$ OF THE OPTIMIZED CONFIGURATIONS ' $C 1$ ' AND ' $C 2$ ' WITH RESPECT TO SNOWFLAKE EQUILIBRIUM OF TCV PULSE 47651 AT $0.8 \mathrm{~s}$.

- $\mathbf{C}_{\mathbf{G s p}}$ the row of the $\mathbf{C}_{\mathbf{G}}$ matrix related to the radial variation of the lower strike point position

- $\alpha_{e}$ the distance of the reference shape from the inner wall at the equatorial plane,

- $\alpha_{s p}$ the distance of the reference lower strike point radial position from the fixed point at $0.73 \mathrm{~m}$ (it is assumed negative if the reference strike point radial position is less than $0.73 \mathrm{~m}$ )

the SF optimization problem consists of finding the maximum value of $q_{c}$ such that the following minimization problem is feasible

$$
\begin{array}{ll}
\min _{\delta \mathbf{I}_{\mathbf{P F}}} & \delta \mathbf{I}_{\mathbf{P F}}^{T} \delta \mathbf{I}_{\mathbf{P F}} \\
& \left\|\mathbf{C}_{\mathbf{G}} \delta \mathbf{I}_{\mathbf{P F}}\right\|<\Delta b \\
& \left\|\mathbf{c}_{\mathbf{G s p}} \delta \mathbf{I}_{\mathbf{P F}}+\alpha_{s p}\right\|<0.01 m \\
& \mathbf{c}_{\mathbf{G e}} \delta \mathbf{I}_{\mathbf{P F}}+\alpha_{e} \geq 0.05 m \\
& s_{i}\left(\overline{\mathbf{I}}_{\mathbf{P F}}+\delta \mathbf{I}_{\mathbf{P F}}\right)<\frac{\bar{s}_{i}}{q_{c}}
\end{array}
$$

Implementing the optimization procedure in (5) on pulse 47651 at $0.8 \mathrm{~s}$, two optimized equilibria were obtained, namely Equilibrium ' $C l$ ' $(\Delta b=0.2 \mathrm{~cm})$ and Equilibrium ' $C 2$ ' $(\Delta b=1 \mathrm{~cm})$. The main parameters of the optimized configurations compared with the snowflake equilibrium of pulse 47651 at $0.8 \mathrm{~s}$ are reported in Table I. The value of the safety factor for the nominal pulse was 1.036 meaning that one of these quantities is within $3.6 \%$ of its limit (vertical force in the F coil support in our case). The optimization of the PF currents in (5) resulted in a safety factor of 1.09 for the ' $C 1$ ' configuration and of 1.20 for the ' $C 2$ ' configuration corresponding to a $9 \%$ and $20 \%$ increase of the allowable plasma current, respectively.

\section{TCV EXPERIMENTAL TESTS ON SNOWFLAKE CONFIGURATIONS}

The proposed optimized equilibria have been successfully tested in TCV pulse 49626. This pulse is organized in four phases:

1) $[0-0.9] \mathrm{s}$ : same as reference pulse 47651 ;

2) $[1.0-1.2] \mathrm{s}$ : after a transient of $0.1 \mathrm{~s}$, impose the $P F$ currents of the Equilibrium ' $C 1$ ' with constant reference plasma current ;

3) $[1.3-1.5] \mathrm{s}$ : after a transient of $0.1 \mathrm{~s}$, impose the PF currents of the Equilibrium ' $C 2$ ' with constant reference plasma current;

4) $[1.5-1.7] \mathrm{s}$ : increase the plasma current by $6 \%$ with a scaling of the PF currents of the Equilibrium ' $C 2$ '.

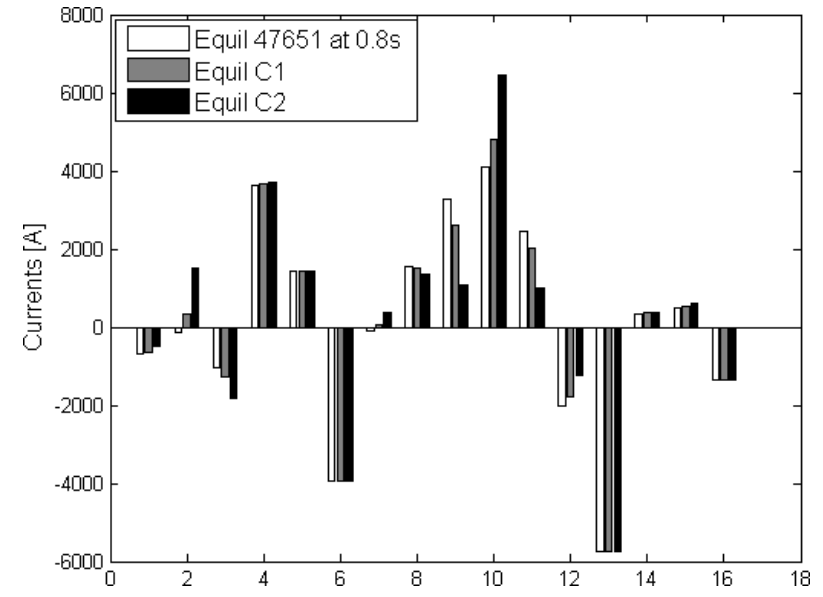

Fig. 6. E1-8 and F1-8 coil currents for the reference SF equilibrium and the optimized configurations $C 1-C 2$.

\begin{tabular}{|c|c|c|c|c|c|c|}
\hline $\mathrm{t}[\mathrm{s}]$ & Shape & $I_{p}[\mathrm{kA}]$ & $l_{i}$ & $\beta_{p}$ & $\mathrm{k}$ & $\gamma\left[s^{-1}\right]$ \\
\hline 0.8 & Eq. 47651 & 240 & 1.04 & 0.18 & 1.82 & 1360 \\
\hline 1.1 & Eq. 'C1' & 240 & 1.02 & 0.17 & 1.80 & 925 \\
\hline 1.5 & Eq. 'C2' & 240 & 0.98 & 0.15 & 1.77 & 610 \\
\hline 1.7 & Eq. 'C2' & 255 & 0.93 & 0.15 & 1.83 & 735 \\
\hline
\end{tabular}

TABLE II

MAIN PLASMA PARAMETER OF TCV PULSE 49626.

The optimized current pattern have been given as a feedforward control action to the current controller which is structured so as to add reference currents to the usual currents coming from the feedback action for the position control.

The reconstruction of the experimental equilibria show minor shape differences with respect to the simulated equilibria ' $C 1$ ' and ' $C 2$ '. These are mainly due to

- the variation of the plasma current profiles. Indeed the configurations ' $C 1$ ' and ' $C 2$ ' have been designed with a constant value of poloidal beta $\beta_{p}=0.12$ and internal inductance $l i=1.00$ while, during the pulse 49626, these values are slightly different from the reference and they move in time ( $l i$ decreasing from 1.04 to $0.93, \beta_{p}$ decreasing from 0.18 to 0.15 );

- the variation of the PF currents due to the feedback on the plasma position, although the feedback correction currents during the four phases were always below $500 \mathrm{~A}$.

The plasma shapes and the main plasma parameters are illustrated in Figure 7 and Table II.

The vertical instability growth rate of the optimized configurations is nearly halved with respect to the reference shape. This can be mainly explained as an effect of the reduced values of the internal inductance (due to the flattening of the current profile). The lowest value of the growth rate does not occur when the internal inductance is minimum only because at $1.5 \mathrm{~s}$ the elongation is 1.77 while at $1.7 \mathrm{~s}$ it increases to 1.83 .

Figures 8-9 show how the optimized currents move the discharge away from the "Eq. 3" safety limit in [17] related 


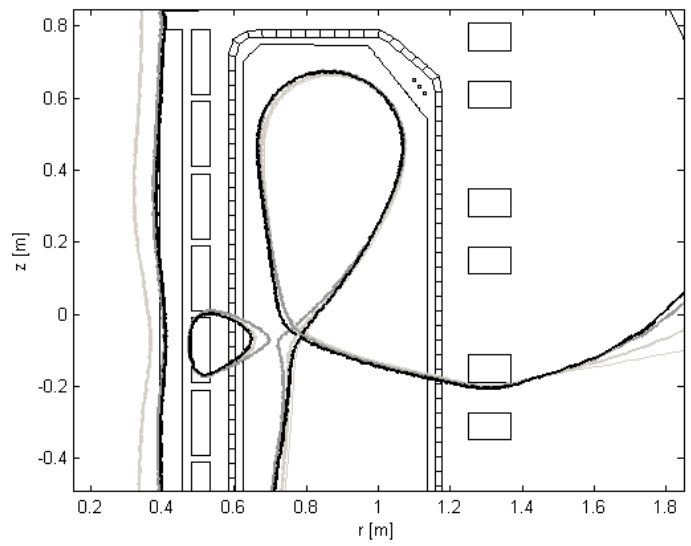

Fig. 7. Sequence of shapes of the TCV pulse 49626 at $0.8 \mathrm{~s}, 1.1 \mathrm{~s}, 1.5 \mathrm{~s}$ and $1.7 \mathrm{~s}$ drawn in gray scale.

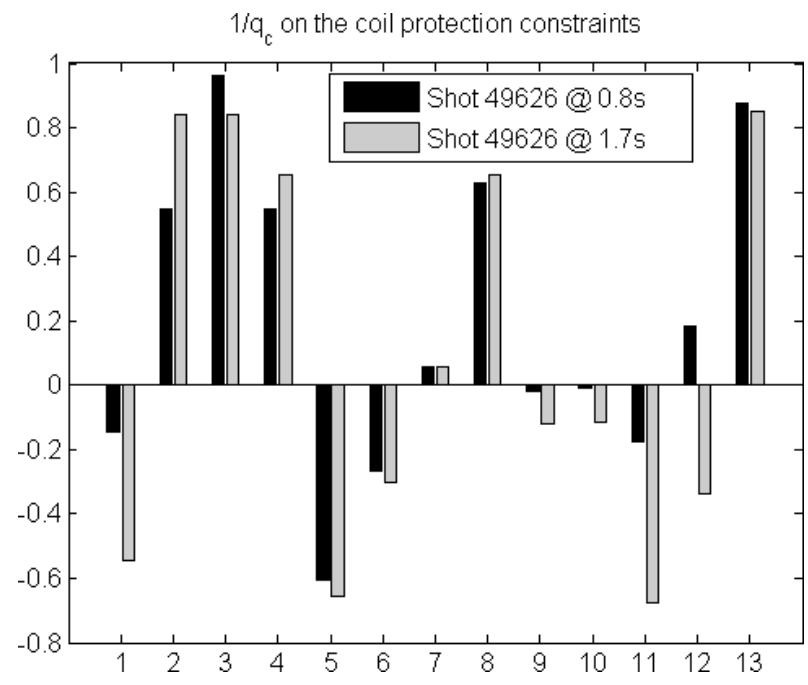

Fig. 8. Safety limits on the coil protection constraints [17].

to the support of the upper F coils:

$\left|0.25 I_{F 1}+0.5 I_{F 2}+I_{F 3}-I_{F 4}-0.5 I_{F 5}-0.25 I_{F 6}\right|<10.5 k A$

which limited the plasma current in the reference configuration at $t=0.8 \mathrm{~s}$. Indeed, the optimized configuration at $t=$ $1.7 \mathrm{~s}$ with the plasma current increased by $6 \%$ is ultimately limited by the absolute current in coil F2 (coil number 10). However, it is important to recognize that the safety factor at $t=1.7 \mathrm{~s}$ is still 1.13 confirming the estimation of the simulation analysis.

In the presented experimental test, the plasma current has been increased by $6 \%$ with a large margin left (theoretically up to a $20 \%$ increase, corresponding to $I p=290 \mathrm{kA}$ ).

Further optimizations may include:

- relaxing the shape constraints;

- taking into account the change of the plasma profiles and the feedback action;

- maximizing other parameters of interest, e.g., flux expansion at the strike points, scrape-off volume, or

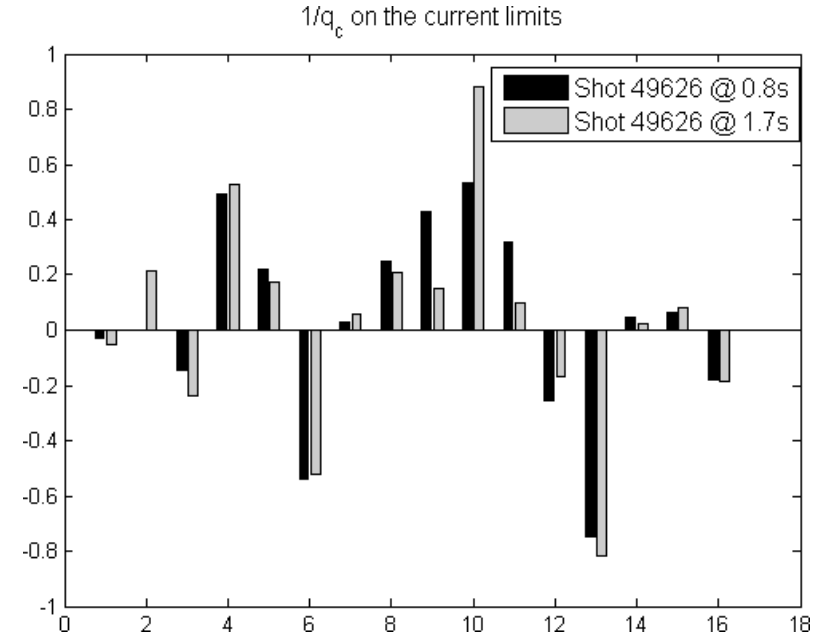

Fig. 9. Safety limits on the [ E-(1:8), F-(1-8)] coil currents.

connection length at the outer side of the scrape-off layer.

\section{CONCLUSIONS}

In this paper the experimental activities carried out in 2013 on TCV to obtain optimized snowflake (SF) configurations have been illustrated. The current optimization procedure developed in [1] has been tested on pulse 47651 where the $\mathrm{PF}$ currents were close to their operational limits with the objective of increasing the plasma current while satisfying the technological constraints and keeping the perturbation of the plasma shape within a tolerance of about $1 \mathrm{~cm}$.

The optimization of the PF currents allowed a $20 \%$ increase of the allowable plasma current while keeping the plasma shape alignment with respect to the nominal 47651 shape within a tolerance of $1 \mathrm{~cm}$.

It is well known that finding distributions of poloidal field currents producing a prescribed plasma surface constitutes an ill posed problem in the sense of Hadamard [19]. This has an impact also for a limited set of PF circuit currents located at fixed positions. Indeed, the linearized relation between the plasma-wall gaps and the PF currents is associated with an ill conditioned output response matrix characterized by a small number of significant singular values [20]. The optimization procedure in [1] takes advantages of this feature in order to optimize the PF currents while fulfilling the TCV technological constraints for a given bound on the tolerable plasma shape changes.

Particular attention has also been paid to the limitation of the growth rate of the vertical instability, which mainly depends on elongation, plasma current profile and plasma position. Indeed, it has been verified that the small plasma shape changes in the optimized configurations did not result in a significant increase of the growth rate.

An experimental validation on pulse 49626 confirmed the calculations with an increase of plasma current limited to $6 \%$ for safety reasons. In the next experimental tests, planned in 2015, further optimizations will be possible, relaxing the 
shape constraints, taking into account the change of the plasma profiles and the feedback action, or maximizing other parameters of interest for the problem of the power exhaust.

\section{ACKNOWLEDGEMENTS}

This activity was carried out under the EFDA Task WP13PEX-02-T03 on experimental investigation of DEMO relevant aspects of the snowflake configuration. This work was partially supported by the European Communities under the Contract of Association between EURATOM and ENEA/CREATE, by the Italian MIUR under PRIN grant 2010SPS9B3 and by the Swiss National Science Foundation.

\section{REFERENCES}

[1] R Albanese, R Ambrosino, and M Mattei. A procedure for the design of snowflake magnetic configurations in tokamaks. Plasma Physics and Controlled Fusion, 56(3):035008, 2014.

[2] F. Romanelli et al. Fusion electicity - a roadmap to the realisation of fusion energy. Technical report, 2012.

[3] D. D. Ryutov. Geometrical properties of a snowflake divertor. Physics of Plasmas, 14(6), 2007.

[4] P.M. Valanju, M. Kotschenreuther, S.M. Mahajan, and J. Canik. Super$\mathrm{x}$ divertors and high power density fusion devices. Physics of Plasmas, 16(5), 2009.

[5] F Piras et al. Snowflake divertor plasmas on TCV. Plasma Phys. Control. Fusion, 51, 2009.

[6] F Piras, S Coda, B P Duval, B Labit, J Marki, S Yu Medvedev, JM Moret, A Pitzschke, O Sauter, and the TCV team. Snowflake divertor experiments on TCV. Plasma Physics and Controlled Fusion, 52(12):124010, 2010.

[7] S. L. Allen et al. Initial snowflake divertor physics studies on DIII-D. In IAEA Fusion Energy Conference, 2012.

[8] Mike Kotschenreuther, Prashant Valanju, Brent Covele, and Swadesh Mahajan. Magnetic geometry and physics of advanced divertors: The X-divertor and the snowflake. Physics of Plasmas, 20(10), 2013.

[9] V.A. Soukhanovskii, R.E. Bell, A. Diallo, S. Gerhardt, S. Kaye, E. Kolemen, B.P. Leblanc, A.G. McLean, J.E. Menard, S.F. Paul, M. Podesta, R. Raman, T.D. Rognlien, A.L. Roquemore, D.D. Ryutov, F. Scotti, M.V. Umansky, D. Battaglia, M.G. Bell, D.A. Gates, R. Kaita, R. Maingi, D. Mueller, and S.A. Sabbagh. Snowflake divertor configuration studies in national spherical torus experiment. Physics of Plasmas, 19(8), 2012.

[10] G.Calabro', R. Albanese, G. Artaserse, R. Ambrosino, F.Crisanti, G. Ramogida, V. Pericoli, Ridolfini, B. Xiao, Y. Guo, and Z. Luo. FAST/EAST snowflake similarity studies. In 7th IAEA Technical Meeting on Steady State Operation of Magnetic Fusion Devices, 2013.

[11] H. Reimerdes, G.P. Canal, B.P. Duval, B. Labit, T. Lunt, W.A.J. Vijvers, S. Coda, G. De Temmerman, T.W. Morgan, F. Nespoli, and B. Tal. Power distribution in the snowflake divertor in TCV. Plasma Physics and Controlled Fusion, 55(12), 2013.

[12] R. Albanese and F. Villone. The linearized CREATE-L plasma response model for the control of current, position and shape in tokamaks. Nuclear Fusion, 38:723-738, 1998.

[13] J.-M. Moret, F. Buhlmann, D. Fasel, F. Hofmann, and G. Tonetti. Magnetic measurements on the TCV tokamak. Review of Scientific Instruments, 69(6):2333-2348, 1998.

[14] F. Hofmann and G. Tonetti. Tokamak equilibrium reconstruction using faraday rotation measurements. Nuclear Fusion, 28(10):1871, 1988.

[15] Alfredo Portone. The stability margin of elongated plasmas. Nuclear Fusion, 45(8):926, 2005.

[16] R. Albanese, R. Ambrosino, and M. Mattei. Efda wp13-pex-02-t03-01 final report on experimental investigation of demo relevant aspects of the snowflake configuration. Technical report, ENEA, 2013.

[17] J M Moret. Signal monitoring of the coil protection of TCV. Internal report, 2003.

[18] F. Hofmann. FBT - a free-boundary tokamak equilibrium code for highly elongated and shaped plasmas. Computer Physics Communications, 48(2):207 - 221, 1988.

[19] K. Lackner. Computation of ideal MHD equilibria. Computer Physics Communications, 12(1):33 - 44, 1976.
[20] M. Ariola and A. Pironti. Plasma shape control for the jet tokamak: An optimal output regulation approach. IEEE Control Systems Magazine, 25(5):65-75, 2005. 